DOI:10.23873/2074-0506-2018-10-1-42-49

Оеновные принципы иммуносупресеивной теџапии после трансплантации почки

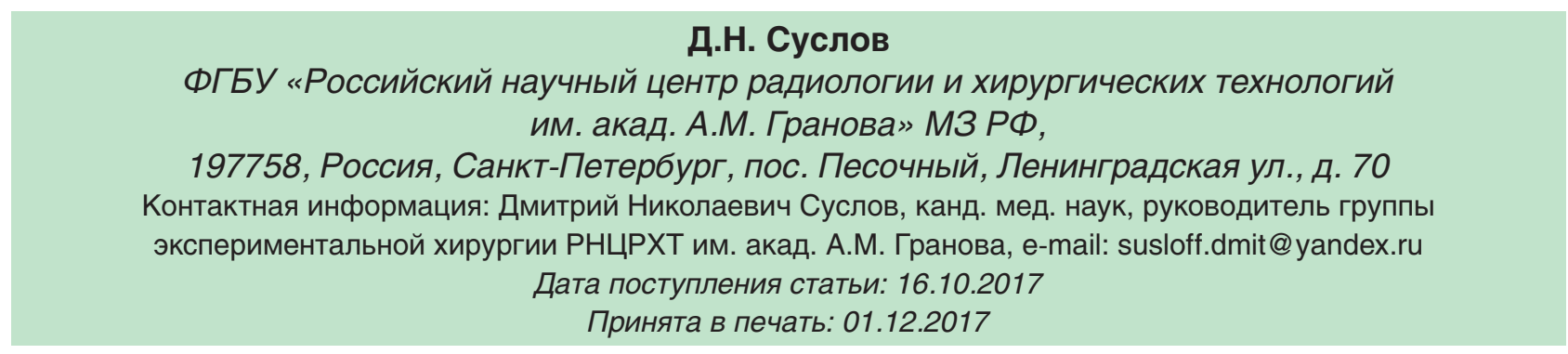

В статъе рассмотрены медицинские и организационные вопросъ индивидуализации имлуносупрессивной терапии у реципиентов почечных аллотрансплантатов. Основой небропротективных схем терапии в последние годъ становятся m-TOR-ингибиторы. Доступность этих препаратов для нуждающихся пациентов представляется недостаточно свободной. В то же время их более иирокое применение в клинической трансплантологии не только повлечет за собой увеличение срока фбункиионирования пересаженной почки, уменъшение рисков развития вирусных инфекций и онкологических заболеваний в отдаленном периоде после пересадки почки, но и позволит рационально использовать бюджетные средства для лечения подобных пациентов.

Ключевые слова: иммуносупрессивная терапия, индивидуализация, доступность, m-TOR-ингибиторы Суслов Д.Н. Основные принципы иммуносупрессивной терапии после трансплантации почки. Трансплантология. 2018;10(1):4249. DOI:10.23873/2074-0506-2018-10-1-42-49

\title{
The basic principles of immunosuppressive therapy after kidney transplantation
}

\section{D.N. Suslov}

Russian Scientific Center of Radiology and Surgical Technologies n.a. acad. A.M. Granov, 70 Leningradskaya Str., Pesochny settlement, St. Petersburg 197758 Russia

Correspondence to: Dmitriy N. Suslov, Cand. Med. Sci., Head of the Experimental Surgery Group, Russian Scientific Center of Radiology and Surgical Technologies n.a. acad. A.M. Granov, e-mail: susloff.dmit@yandex.ru

Received: 16 October 2017

Accepted for publication: 1 December 2017

The article deals with medical and organizational issues of individually-adapted immunosuppressive therapy in renal allograft recipients. In the recent years, the basis of nephroprotective therapy schemes includes $m$-TOR inhibitors. The accessibility of these drugs for those in need is limited. Meanwhile, a more widespread use of these drugs in clinical transplantation would both increase the life span of a transplanted kidney, and reduce the risk of viral infections and cancer in the long-term after kidney transplantation, and would also allow a rational use of budget funds for the treatment of such patients.

Keywords: immunosuppressive therapy, individual adaptation, accessibility, m-TOR inhibitors

Suslov D.N. The basic principles of immunosuppressive therapy after kidney transplantation. Transplantologiya. The Russian Journal of Transplantation. 2018;10(1):42-49. (In Russian). DOI:10.23873/2074-0506-2018-10-1-42-49

ВЗН - высокозатратные нозологии

ДЛО - дополнительное лекарственное обеспечение

ИСТ - иммуносупрессивная терапия

ИКН - ингибиторы кальцинейрина

КС - кортикостероиды

МФК - микофеноловая кислота

Ня - нежелательное явление
ОНЛС - обеспечение необходимыми лекарственными средствами

СКФ - СКорость клубочковой фильтрации

TAK - такролимус

ЦСА - циклоспорин

ЭВЕ - эверолимус 


\section{PROBLEMATIG ASPEGTS}

Одним из значимых компонентов лечения пациентов методом пересадки почки является послеоперационная иммуносупрессивная терапия (ИСТ). Начинается такая терапия в ряде случаев еще в период подготовки реципиента к операции и продолжается весь срок фрункционирования почечного, печеночного, сердечного, легочного и иных трансплантатов и пересаженных комплексов фрактически пожизненно. В отдаленном периоде ИСТ становится тем фрактором, который во многом определяет продолжительность функционирования пересаженного органа и жизни реципиента [1].

Схематично ИСТ с момента операции может быть представлена как комбинация следующих препаратов: селективные иммунодепрессанты и (или) ингибитор интерлейкина + ингибиторы кальцинейрина $($ ИКН) + селективные иммунодепрессанты или другие иммунодепрессанты + кортикостероиды (КС) системного действия. То есть с момента операции ИСТ является четырехкомпонентной или, в ряде случаев, трехкомпонентной. ИСТ после выписки пациента на амбулаторное лечение: также комбинированная терапия, в подавляющем большинстве случаев - трехкомпонентная. Ее схема практически такая же, как и в раннем послеоперационном периоде: ИКН в сочетании с селективными иммунодепрессантами или другими иммунодепрессантами и $\mathrm{KC}$ системного действия. В дальнейшем ИСТ может изменяться в зависимости от состояния пересаженного органа и реципиента. При этом набор препаратов остается практически неизменным на протяжении всего срока фрункционирования трансплантата, а смена препаратов осуществляется только в соответствии со строго определенными медицинскими показаниями. Как уже было сказано, наиболее часто используют трехкомпонентные схемы, реже - двухкомпонентные и монотерапию. Доля пациентов с фрункционирующим почечным трансплантатом, получающих двухкомпонентную ИСТ, в Санкт-Петербурге составляет 14,1\% (данные на начало 2017 г.). Монотерапию применяют в основном у больных с фрунццинирующим печеночным трансплантатом.

Из используемых схем наибольшее распространение в последние годы получила схема, включающая различные лекарственные фрормы ИКН такролимуса (ТАК), один из препаратов микофеноловой кислоты (МФК) и КС (или без них). Подобную терапию применяют после выписки из стационара у 59,42\% пациентов, другой ИКН, циклоспорин (ЦсА), используют у $35,68 \%$ больных в сочетании с одним из препаратов МФК и $\mathrm{KC}$, и только у 4,9\% больных удается отказаться от применения ИКН.

В табл. 1 представлены препараты, используемые для ИСТ в Российской Федерации, и профили их нежелательных явлений (НЯ) [2-4].

Табл. 1 наглядно демонстрирует отсутствие идеального препарата и, следовательно, отсутствие идеальной комбинации препаратов в настоящее время. У всех лекарственных средств наблюдаются побочные эфрфекты или НЯ. При этом препараты из разных групп могут иметь общие НЯ, которые могут проявиться с большей вероятностью при их комбинировании.

Таблица 1. Полуколичественное сравнение профилей побочных эффектов иммуносупрессивных препаратов Table 1. A semi-quantitative comparison of adverse effect profiles of immunosuppressants

\begin{tabular}{|c|c|c|c|c|c|c|c|}
\hline НЯ / Препарат & ЦсА & TAK & МФК & Азатиоприн & Белатасепт & KC & m-TOR-ингибиторы \\
\hline Нефрротоксичность & +++ & +++ & - & - & - & - & - \\
\hline Нейротоксичность & ++ & +++ & - & - & + & + & - \\
\hline Гипертензия & +++ & ++ & - & - & - & +++ & - \\
\hline Гиперлипидемия & ++ & + & - & - & - & ++ & +++ \\
\hline Диабет & + & +++ & - & - & - & ++ & -+ \\
\hline Гепатотоксичность & + & + & - & + & - & - & + \\
\hline Гиперплазия десен & ++ & - & - & - & - & - & - \\
\hline Гирсутизм & ++ & - & - & - & - & + & - \\
\hline Диарея & + & + & +++ & + & ++ & - & +++ \\
\hline Лейкопения & - & - & +++ & +++ & + & - & + \\
\hline Тромбоцитопения & - & - & + & + & + & - & ++ \\
\hline $\begin{array}{l}\text { Инфекции / посттрансплантационное } \\
\text { лимфопролиферативное заболевание }\end{array}$ & + & + & + & + & +++ & + & + \\
\hline
\end{tabular}


Использование ИКН (ЦсА, ТАК) позволило достичь 90\% годичной выживаемости трансплантатов, снизить до $5 \%$ частоту острого отторжения в раннем посттрансплантационном периоде, однако при продолжительном применении оно порождает серьезные $\mathrm{HЯ} \mathrm{-} \mathrm{недрротоксичность} \mathrm{и} \mathrm{гепа-}$ тотоксичность, которые могут реализовываться независимо от доз препаратов. Это послужило одной из причин того, что в отдаленном периоде было достигнуто весьма незначительное улучшение периода полужизни почечного трансплантата: с 6,8 года в 1989 г. до 8 лет в 1995 г. и 8,4 года в 2000 г. [5-7].

Основным принципом ИСТ в настоящее время, на взгляд одного из авторов, становится ее индивидуальность, т.е. при подборе такой терапии необходимо учитывать все преимущества и недостатки назначаемых препаратов у каждого конкретного пациента [8]. Однако реализовать принцип индивидуальности можно только при соблюдении следующих положений: при применении эффрективных и безопасных препаратов, при продолжении терапии без изменений после выписки больного из стационара, - т.е. должен присутствовать принцип преемственности; при этом назначаемый в трансплантационном центре препарат должен быть в свободном доступе для пациента. Таким образом, основные принципы ИСТ в порядке последовательности могут быть определены как:

1. Эффрективность.

2. Безопасность.

3. Преемственность.

4. Доступность.

5. Индивидуальность.

Принципы эффеективности и безопасности имеют решающее значение, так как практически все схемы включают препараты, которые могут быть отнесены к препаратам «узкого терапевтического диапазона», а такие препараты, как ЦсА и ТАК, - к препаратам «критичной дозы» [1].

Определение эффективности и безопасности лекарственных препаратов приведены в статье 4 Федерального закона «Об обращении лекарственных средств»: безопасность лекарственных средств - характеристика лекарственных средств, основанная на сравнительном анализе их эфффективности и оценки риска причинения вреда здоровью. Эффрективность лекарственных средств - характеристика степени положительного влияния лекарственных средств на течение болезни [9]. В случае медикаментозного лечения реципиента органного трансплантата эффектив- ность может быть определена как применение препаратов или комбинации препаратов, которые способствуют достижению стоящей перед врачом цели.

Преемственность относится к порядку последования, замещения препаратов [10]. В нашем случае - это возможность продолжать лечение по месту жительства реципиента теми же препаратами (по международному непатентованному наименованию и по торговому названию), которыми было начато лечение в центре трансплантации, возможность безопасной замены одного препарата на другой.

Доступность - от «доступный», т.е. нескрытый, дающий свободный доступ [10]. В нашем случае это возможность свободного получения каждым конкретным пациентом необходимого препарата по показаниям.

В 2011 г. была принята Федеральная программа обеспечения необходимыми лекарственными средствами (ОНЛС). Программа преследовала следующие цели: развитие системы адресной социальной поддержки населения; осуществление перехода на новый порядок финансирования мер по социальной поддержке льготных категорий населения; реализация мер по повышению доступности и качества медицинской и лекарственной помощи; внедрение методов стандартизации медицинской помощи; совершенствование контрольно-надзорных мероприятий [11]. Реализация программы ОНЛС обеспечивает необходимыми препаратами пациентов, получающих пожизненную ИСТ. Лекарственные препараты, необходимые для проведения терапии, больные получают по программе дополнительного лекарственного обеспечения (ДЛО) и по программе лечения «Семь высокозатратных нозологий»- ВЗН («7 нозологий»). Основные иммуносупрессивные препараты включены в перечень для лечения ВЗН, по программе ДЛО возможно получение препаратов, предназначенных главным образом для лечения осложнений ИСТ. При этом по программе «7 нозологий» закупают 5 препаратов, средняя цена за 1 мг составляет 34 рубля 96 копеек. По программе ДЛО пациенты могут получить 6 препаратов, средняя цена за 1 мг которых составляет 63 рубля 53 копейки. При этом стоимость одного рецепта по программе «7 нозологий», определенная Правительством Российской Федерации, составляет 30095 рублей, а на одного больного для реализации программы ДЛО из федерального бюджета выделяют 870 рублей в месяц. 


\section{PROBLEMATIC ASPEGTS}

Следует отметить, что программа ОНЛС не смогла обеспечить индивидуализацию ИСТ. Выполнение данной программы способствовало постепенному сокращению доли оригинальных препаратов и росту числа воспроизведенных препаратов на рынке препаратов для ИСТ. В настоящее время по программе «7 нозологий» пациенты могут получить только 3 оригинальных препарата, при этом одна оригинальная лекарственная форма может быть использована только у больных с ограниченными показаниями.

Именно применение воспроизведенных иммуносупрессивных препаратов и увеличение числа доноров с расширенными критериями сделали в России востребованными схемы ИСТ, которые не оказывают повреждающего действия на пересаженную почку, - т.е. нефропротективные схемы ИСТ.

Заслуживают внимания следующие данные. Через 12 месяцев после трансплантации почки скорость клубочковой фрильтрации - СКФ (MDRD) составляет 59,08 + 18,91 мл/мин/1,73 м² у пациентов, получавших терапию с использованием схемы ТАК + МФК + КС. У больных, получавших схему ИСТ на основе ЦсА: ЦсА + MФК + KC - CKФ (MDRD) через 12 месяцев составила $62,43+20,23$ мл/мин/1,73 м². Доля пациентов с СКФ менее 40 мл/мин в данных группах составила $16 \%$ и $26,92 \%$ соответственно. Для сравнения: у 2 реципиентов, оперированных в 1987 и 1988 гг., у которых ИКН были отменены спустя 18-24 месяца после операции, CKФ (MDRD) через 27 и 28 лет после пересадки почки составила 51,28 и 61,24 мл/мин/1,73 м² соответственно.

Кроме того, в раннем и отдаленном периодах после пересадки почки перед пациентом и его лечащим врачом возникают разные риски, в отдаленном периоде усиливается влияние ИСТ, особенно отдельных препаратов, используемых для терапии. Если в раннем периоде после пересадки почки основными рисками выступают острое отторжение почечного трансплантата, отсроченная или сниженная функция трансплантата, заживление послеоперационной раны, то проводимая нами терапия должна предупреждать развитие отторжения, не усугублять имеющиеся нарушения фрункции трансплантата и не препятствовать восстановлению его функции [12]. Схема с использованием ИКН в сочетании с препаратами $\mathrm{MФК} \mathrm{и} \mathrm{КС} \mathrm{представляется} \mathrm{оптимальной} \mathrm{для}$ применения в раннем периоде после пересадки почки. Риски отдаленного периода после трансплантации почки - это прежде всего риски, свя- занные с использованием нефротоксичных препаратов, вплоть до развития интерстициального фиброза и тубулярной атрофрии, риски развития и прогрессирования вирусной инфекции, вызванной вирусом цитомегалии, а также опасность развития онкологических заболеваний [12]. Нужно ли при возникновении данных НЯ или осложнений менять терапию? Безусловно, нужно. Нужно ли предотвращать или отдалять по времени появление этих НЯ и осложнений? Безусловно нужно. И здесь становится актуальной двухэтапная схема ИСТ, предложенная испанскими трансплантологами [12]: в раннем периоде - ИСТ с использованием ИКН; в отдаленном периоде - нефропротективные схемы ИСТ.

Одним из компонентов нефропротективных схем выступают препараты из группы ингибиторов мишени для рапамицина у млекопитающих (mammalian target of rapamycin, m-TOR). Подавление m-TOR приводит к иммуносупрессивному эфоректу, блокирует пролиферацию и ангиогенез, затрудняет репликацию вируса цитомегалии, в конечном итоге предупреждает развитие хронической трансплантационной недропатии, препятствует росту опухолевых клеток [13-19]. Ингибиторы m-TOR применяют в сочетании с ИКН и $\mathrm{KC}$, без ИКН в сочетании с солями МФК и КС; это позволяет эфрфективно и безопасно использовать схемы ИСТ без КС. Назначение m-TOR-ингибиторов возможно в разные сроки после трансплантации: сразу после операции в сочетании с ИКН и $\mathrm{KC}$, от 3 до 6 месяцев после операции с сохранением низких доз ИКН или при наличии показаний и возможности с отменой ИКН - ранняя конверсия, или добавление их в схему ИСТ на любых сроках после операции - поздняя конверсия [20, 21]. Наличие в свободном доступе на рынке препаратов из группы m-TOR-ингибиторов, позволяющих эфрфективно и безопасно использовать новые комбинации иммуносупрессивных препаратов, послужит хорошей основой для дальнейших работ по индивидуализации ИСТ.

В настоящее время доступным препаратом из группы m-TOR-ингибиторов выступает эверолимус (ЭВЕ). Препарат включен в список жизненно необходимых препаратов, а также в список препаратов, закупка которых осуществляется по программе ДЛО. Второй препарат из данной группы - сиролимус, первый по времени появления на рынке и по началу использования у реципиентов почечных аллотрансплантатов, - не включен в перечень жизненно необходимых препаратов, 
его доступность для пациентов представляется недостаточно свободной, он может быть получен только при наличии медицинских показаний в сочетании с желанием и волей специалиста, его назначающего. При этом по цене за 1 мг препарата ЭВЕ является наиболее дорогим из иммуносупрессивных препаратов - 292,67 рубля. Схемы ИСТ с использованием ЭВЕ стоят не дороже схем с традиционно используемыми препаратами. Данные о стоимости месячного и годичного лечения реципиентов почечного аллотрансплантата приведены в табл. 2.

Таблица 2. Стоимость иммуносупрессивной терапии в отдаленном периоде после пересадки почки

Table 2. The cost of immunosuppressive therapy in the long-term after kidney transplantation

\begin{tabular}{|l|c|c|}
\hline \multicolumn{1}{|c|}{ Схема ИСТ } & $\begin{array}{c}\text { Стоимость } \\
\text { лечения } \\
\text { (1 месяц), } \\
\text { руб. }\end{array}$ & $\begin{array}{c}\text { Стоимость } \\
\text { лечения } \\
\text { (12 месяцев), } \\
\text { руб. }\end{array}$ \\
\hline ЦСА + МФК + КС & 15880,46 & 190565,52 \\
\hline ТАК + МФК + КС & 19573,47 & 234881,64 \\
\hline ТАК (пролонг.) + МФК + КС & 20701,91 & 248422,92 \\
\hline ЭВЕ + ЦСА + КС & 12087,63 & 145050,36 \\
\hline ЭВЕ + МФК + КС & 25509,56 & 306114,72 \\
\hline ЭВЕ + ТАК (пролонг.) + КС & 22386,88 & 268642,56 \\
\hline
\end{tabular}

Данные, приведенные в табл. 2, наглядно показывают, что индивидуализация ИСТ не только не значимо увеличивает стоимость лечения пациентов с фрункционирующим почечным аллотрансплантатом, но и позволяет рационально использовать бюджетные средства, выделяемые на эти цели. Наиболее дорогая схема - ЭВЕ + $\mathrm{M} \Phi \kappa+\mathrm{KC}$, но число больных, получающих такое лечение, невелико. Ни одна из представленных в таблице схем не превышает стоимость рецепта, определенную Правительством РФ (стоимость одного рецепта равна стоимости месячного лечения). В последние годы в Санкт-Петербурге у $16 \%$ пациентов, перенесших трансплантацию солидных органов, используют m-TOR-ингибиторы. И только $40 \%$ реципиентов, перенесших трансплантацию почки, из числа тех, кто получает m-TOR-ингибиторы, не получают ИКH, т.е. им проводят лечение по схеме ЭВЕ + MФК + КС.

HЯ на фоне терапии m-TOR-ингибиторами в большинстве своем являются дозозависимыми и хорошо поддаются медикаментозной терапии или купируются после замены одного препарата группы m-TOR-ингибиторов на другой [12].

Еще одной значимой проблемой в отдаленном периоде выступает несоблюдение терапевтического режима. Несмотря на высокую частоту отмены препарата после инициации лечения, связанную с побочными действиями, однократный прием m-TOR-ингибитора сиролимуса может оказывать положительное влияние на долгосрочное соблюдение режима. Имеются ограниченные данные по улучшению соблюдения режима терапии при приеме комбинации сиролимус + ТАК пролонгированного действия в низкой дозе [22].

На основании имеющихся данных можно утверждать, что, хотя ИКН эфрфективно минимизируют риск острого отторжения трансплантата, они имеют ряд ограничений. Режимы иммуносупрессии на основе сиролимуса без ИКН или со сниженной дозой ИКН позволяют в большей степени сохранить почечную функцию, снизить риск новообразований и инфекций [23]. «Золотым стандартом» ИСТ в начальном периоде являются ИКН, в то время как основной поддерживающей ИCT могут выступать m-TOR-ингибиторы сиролимус и ЭВЕ [12, 21, 22].

По нашему мнению, препараты группы m-TOR-ингибиторов могут и должны быть использованы у: пациентов с низким или умеренным иммунологическим риском; у больных молодого возраста с целью обеспечения хорошей длительной функции почечного трансплантата; у реципиентов старше 50 лет, получающих трансплантаты от субоптимальных доноров, имеющих в личном или семейном анамнезе сердечно-сосудистую патологию, инфаркты, инсульты, онкологические заболевания, не имеющих или имеющих протеинурию не более 0,8 г/сут, у которых нет выраженных нарушений в уровне липидов сыворотки крови (гиперхолестеринемия - не более 9 ммоль/л и гипертриглицеридемия - не более 8,5 ммоль/л); пациентов с индексом массы тела менее 35. При назначении этих препаратов необходимо учитывать наличие и степень выраженности возможных противопоказаний, таких как протеинурия более 1 г/сут, рефрактерная дислипидемия, рецидивирующий или вновь приобретенный гломерулонефрит, острое или хроническое отторжение, планируемое оперативное вмешательство или наличие хирургических ран на стадии заживления, беременность или желание пациентки забеременеть. 


\section{ПРОБПЕМНЫЕ АЯПЕНТЫ}

\section{PROBLEMATIG ASPEGTS}

\section{Заключение}

Индивидуализация пожизненной ИСТ выступает в качестве основного фрактора отдаленного периода после трансплантации того или иного органа, который определяет срок функционирования трансплантата и, соответственно, жизни реципиента. Подбор индивидуальной ИСТ для каждого конкретного пациента должен происходить на основании только медицинских критериев при наличии в свободном доступе эфффективных и безопасных иммуносупрессивных препаратов. Индивидуальный подход к медикаментозной терапии позволит оптимизировать затраты на наблюдение и лечение больных методом трансплантации в отдаленном периоде после операции, а также добиться максимальной медицинской и социальной реабилитации реципиентов органных трансплантатов.

\section{Выводы}

1. Индивидуализация пожизненной иммуносупрессивной терапии позволяет добиться максимальной медицинской и социальной реабилитации реципиентов органных трансплантатов и увеличивает срок функционирования пересаженного органа.
2. Индивидуализация иммуносупрессивной терапии возможна только при наличии в свободном доступе эффективных и безопасных препаратов.

3. «Идеального» иммуносупрессивного препарата и «идеальной» схемы иммуносупрессивной терапии в настоящее время нет. «Идеальной» схема иммуносупрессивной терапии может быть только для каждого конкретного пациента после тщательного, взвешенного подбора.

4. Выбор и сочетание препаратов для иммуносупрессивной терапии должны определяться только показаниями и противопоказаниями к их применению. Ценовые и иные «немедицинские» факторы должны только приниматься во внимание, а не определять целесообразность использования препарата в клинической практике.

5. Все зарегистрированные в стране иммуносупрессивные препараты должны быть внесены в список «жизненно необходимых», закупку всех препаратов для продолжения высокотехнологичного лечения целесообразно осуществлять за счет одного источника финансирования.

6. Индивидуальный подход к медикаментозной терапии позволит оптимизировать все затраты на наблюдение и лечение пациентов методом трансплантации в отдаленном периоде после операции.

КОНФЛИКТ ИНТЕРЕСОВ. АвторЫ заяВЛЯЮт Об отСутСтвИи КОНфЛикта интересов. CONFLICT OF INTERESTS. Authors declare no conflict of interest.

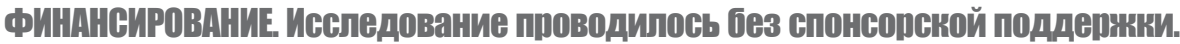
FINANGING. The study was performed without external funding. 


\section{Литература}

1. Лекарственный мониторинг и взаимозаменяемость оригинальных и генерических иммунодепрессантов с узким терапевтическим индексом. Национальные клинические рекомендации. M., 2014. 23 c.

2. Kuypers D.R. Benefit-risk assessment of sirolimus in renal transplantation. Drug Saf. 2005;28(2):153-181. PMID:15691225

3. Halloran P.F. Immunosuppressive drugs for kidney transplantation. N Engl. J. Med. 2004;351(26):2715-2729 PMID:15616206 https://doi.org/10.1056/ NEJMra033540

4. Heemann U., Viklicky O. The role of belataceptin transplantation: results and implications of clinical trials in the context of other new biological immunosuppressant agents. Clin. Transplant. 2013;27(1):E3-11. PMID:23199344 DOI: $10.1111 /$ ctr. 12044

5. Opelz G., Dohler B. Influence of Immunosuppressive Regimens on Graft Survival and Secondary Outcomes after Kidney Transplantation. Transplantation. 2009;87(6):795-802. PMID:19300179 DOI:10.1097/TP.0b013e318199c1c7

6. Morris P.J. Transplantation - a medical miracle of the 20 th century. N. Engl. J. Med. 2004;351(26):26782680. PMID:15616201 DOI:10.1056/ NEJMp048256

7. Campbell S., McDonald S., Chang S et al. Chapter 8. Transplantation. In: McDonald S., Chang S., Excell L., eds. ANZDATA registry report 2007. Adelaide, South Australia: Australia and New Zealand Dialysis and Transplant Registry, 2007.

8. Jain K.K. From molecular diagnostics to personalized medicine. The IBC Workshop, London, UK, 1st May, 2002. Exp. Rev. Mol. Diagn. 2002;2(4):299-301. DOI:10.1586/14737159.2.4.299
9. Об обращении лекарственных средств. Федеральный закон от 12.04.2010 № 61Ф3. Ред. 25.11.2013. URL: https://normativ. kontur.ru/document? moduleId$=1 \&$ documentId $=304074$

10. Даль В.И. Толковый словарь живого великорусского языка: в 4 т. М.: Русский язык, 1978-1980.

11. Юргель Н.В., Тельнова Е.А. ДЛО ОНЛС, «7 нозологий», а что дальше? Вестник Росздравнадзора. 2008;(5):421. URL: http://www.remedium.ru/ public/journal/vr/2008/VR_05_2008.pdf 12. Pascual J., Boletisb J.N., Campistolc J.M. Everolimus (Certican) in renal transplantation: a review of clinical trial data, current usage, and future directions. Transplant. Rev. 2006;20(1):1-18. DOI:10.1016/j.trre.2005.10.005

13. Ormiston J.A., Serruys P.W., Regar E. et al. A bioabsorbable everolimus-eluting coronary stent system for patients with single de-novo coronary artery lesions (ABSORB) a prospective open-label trial. Lancet. 2008;371(9616):899-907. PMID:18342684 DOI:10.1016/S01406736(08)60415-8

14. Ponticelli C. Can mTOR inhibitors reduce the risk of late kidney allograft failure? Transpl. Int. 2008;21(1):2-10. PMID:17635837 DOI:10.1111/j.14322277.2007.00524.x

15. Cruzado J.M. Nonimmunosuppressive effects of mammalian target of rapamycin inhibitors. Transplant. Rev. (Orlando). 2008;22(1):73-81. PMID:18631860 DOI:10.1016/j.trre.2007.09.003

16. Contreras A.G., Dormond O., Edelbauer M. et al. mTOR-understanding the clinical effects. Transplant. Proc. 2008;40(10, Suppl.):S9-S12. PMID:19100913 DOI:10.1016/j.transproceed.2008.10.011

17. Hudes G.R. Targeting mTOR in renal cell carcinoma. Cancer. 2009;115(10, Suppl.):2313-2320. PMID:19402072 DOI: $10.1002 /$ cncr. 24239

18. Eisen H. Long-term cardiovascular risk in transplantation--insights from the use of everolimus in heart transplantation. Nephrol. Dial. Transplant. 2006;21(Suppl. 3):iii9-13. PMID:16815858 DOI: $10.1093 / \mathrm{ndt} / \mathrm{gfl} 295$

19. Schuler W., Sedrani R., Cottens S. et al. SDZ RAD, a new rapamycin derivative: pharmacological properties in vitro and in vivo. Transplantation. 1997;64(1):36-42. PMID:9233698

20. Guba M., Pratschke J., Hugo C. et al. Early conversion to a sirolimus-based, calcineurin-inhibitor-free immunosuppression in the SMART trial: observational results at 24 and 36 months after transplantation. Transplant. Int 2012;25(4):416-423. PMID:22320241 DOI:10.1111/j.1432-2277.2012.01432.x

21. Guba M., Pratschke J., Hugo C. et al. Renal function, efficacy, and safety of sirolimus and mycophenolate mofetil after short-term calcineurin inhibitorbased quadruple therapy in de novo renal transplant patients: one-year analysis of a randomized multicenter trial. Transplantation. 2010;90(2):175183. PMID:20463641 DOI:10.1097/ TP.0b013e3181e11798

22. Jun H., Kim M.G., Jung C.W. Clinical advantages including medication adherence with conversion to oncedaily advagraf and sirolimus combination in stable kidney recipients. Int. J. Clin. Pharmacol. Ther. 2016;54(2):81-86. PMID:26709601 DOI:10.5414/CP202518. 23. Hariharan S., McBride M.A., Cherikh W.S. et al. Post-transplant renal function in the first year predicts long-term kidney transplant survival. Kidney Int. 2002;62(1):311-318. PMID: 12081593 DOI:10.1046/j.1523-1755.2002.00424.x 


\section{References}

1. Drug monitoring and interchangeability of original and generic, immunodepressive drugs with narrow therapeutic index. National clinical guidelines. Moscow, 2014. 23 p.

2. Kuypers D.R. Benefit-risk assessment of sirolimus in renal transplantation. Drug Saf. 2005;28(2):153-181. PMID:15691225

3. Halloran P.F. Immunosuppressive drugs for kidney transplantation. $N$ Engl J Med. 2004;351(26):2715-2729. PMID:15616206 DOI:10.1056/NEJMra033540

4. Heemann U., Viklicky O. The role of belataceptin transplantation: results and implications of clinical trials in the context of other new biological immunosuppressant agents. Clin Transplant. 2013;27(1):E3-11. PMID:23199344 DOI: $10.1111 /$ ctr. 12044

5. Opelz G., Dohler B. Influence of Immunosuppressive Regimens on Graft Survival and Secondary Outcomes after Kidney Transplantation. Transplantation. 2009;87(6):795-802. PMID:19300179 DOI:10.1097/TP.0b013e318199c1c7

6. Morris P.J. Transplantation - a medical miracle of the 20th century. $N$ Engl J Med. 2004;351(26):2678-2680. PMID:15616201 DOI:10.1056/NEJMp048256

7. Campbell S., McDonald S., Chang S., et al. Chapter 8. Transplantation. In: McDonald S., Chang S., Excell L., eds. ANZDATA registry report 2007. Adelaide, South Australia: Australia and New Zealand Dialysis and Transplant Registry, 2007.

8. Jain K.K. From molecular diagnostics to personalized medicine. The IBC Workshop, London, UK, 1st May, 2002. Exp Rev Mol Diagn. 2002;2(4):299-301. DOI:10.1586/14737159.2.4.299

9. On the circulation of medicines. Federal Law of 12.04.2010, № 61FL. November 25, 2013 ed. Available at: https://
normativ.kontur.ru/document?moduleI $\mathrm{d}=1 \&$ documentId $=304074$

10. Dal' V.I. Explanatory dictionary of the living Great Russian language: in 4 volumes. Moscow: Russkiy yazyk Publ., 1978-1980.

11. Yurgel' N.V., Tel'nova E.A. ADS PNM, "7 nosologies," and then what? Vestnik Roszdravnadzora. 2008;(5):4-21. Available at: http://www.remedium.ru/ public/journal/vr/2008/VR_05_2008.pdf 12. Pascual J., Boletisb J.N., Campistolc J.M. Everolimus (Certican) in renal transplantation: a review of clinical trial data, current usage, and future directions. Transplant Rev. 2006;20(1):1-18. DOI: $10.1016 /$ j.trre.2005.10.005

13. Ormiston J.A., Serruys P.W., Regar E., et al. A bioabsorbable everolimus-eluting coronary stent system for patients with single de-novo coronary artery lesions (ABSORB) a prospective open-label trial. Lancet. 2008;371(9616):899-907. PMID:18342684 DOI:10.1016/S01406736(08)60415-8

14. Ponticelli C. Can mTOR inhibitors reduce the risk of late kidney allograft failure? Transpl Int. 2008;21(1):2-10. PMID:17635837 DOI:10.1111/j.14322277.2007.00524.x

15. Cruzado J.M. Nonimmunosuppressive effects of mammalian target of rapamycin inhibitors. Transplant Rev. (Orlando). 2008;22(1):73-81. PMID:18631860 DOI: $10.1016 /$ j.trre.2007.09.003

16. Contreras A.G., Dormond O., Edelbauer M., et al. mTOR-understanding the clinical effects. Transplant Proc. 2008;40(10 Suppl):S9-S12. PMID:19100913 DOI:10.1016/j.transproceed.2008.10.011

17. Hudes G.R. Targeting mTOR in renal cell carcinoma. Cancer. 2009;115(10 Suppl):2313-2320. PMID: 19402072 DOI:10.1002/cncr.24239
18. Eisen H. Long-term cardiovascular risk in transplantation--insights from the use of everolimus in heart transplantation. Nephrol Dial Transplant. 2006;21(Suppl 3):iii9-13. PMID:16815858 DOI:10.1093/ndt/gfl295

19. Schuler W., Sedrani R., Cottens S., et al. SDZ RAD, a new rapamycin derivative: pharmacological properties in vitro and in vivo. Transplantation. 1997;64(1):36-42. PMID:9233698

20. Guba M., Pratschke J., Hugo C., et al. Early conversion to a sirolimus-based, calcineurin-inhibitor-free immunosuppression in the SMART trial: observational results at 24 and 36 months after transplantation. Transplant Int. 2012;25(4):416-423. PMID:22320241 DOI: $10.1111 / \mathrm{j} .1432-2277.2012 .01432 . \mathrm{x}$

21. Guba M., Pratschke J., Hugo C, et al. Renal function, efficacy, and safety of sirolimus and mycophenolate mofetil after short-term calcineurin inhibitorbased quadruple therapy in de novo renal transplant patients: one-year analysis of a randomized multicenter trial. Transplantation. 2010;90(2):175183. PMID:20463641 DOI:10.1097/ TP.0b013e $3181 \mathrm{e} 11798$

22. Jun H., Kim M.G., Jung C.W. Clinical advantages including medication adherence with conversion to oncedaily advagraf and sirolimus combination in stable kidney recipients. Int $J$ Clin Pharmacol Ther. 2016;54(2):81-86. PMID:26709601 DOI:10.5414/CP202518. 23. Hariharan S., McBride M.A., Cherikh W.S., et al. Post-transplant renal function in the first year predicts long-term kidney transplant survival. Kidney Int. 2002;62(1):311-318. PMID: 12081593 DOI:10.1046/j.1523-1755.2002.00424.x 